\title{
Reducing Time Delays in Computing Numerical WeATHER MODELS AT REgIONAL AND LOCAL LEVELS: A GRID-BASED APPROACH
}

\author{
Eugenio Sper de Almeida ${ }^{1,2}$, Michael Bauer ${ }^{2}$ \\ ${ }^{1}$ Center for Weather Forecast and Climate Studies, National Institute for Space Research, \\ Cachoeira Paulista, SP, Brazil \\ eugenio.almeidaecptec.inpe.br \\ ${ }^{2}$ Department of Computer Science, The University of Western Ontario, \\ London, ON, Canada \\ bauer@uwo. ca
}

\begin{abstract}
This paper introduces a grid-based approach to reduce the time to deliver local meteorological forecast information. The computation of local meteorological forecasts relies on the execution of both regional and local scale Numerical Weather Prediction models. Typically, such models require extensive computation on large compute clusters. Moreover, the models are commonly executed sequentially, since local models rely on the output of a regional model. The approach introduced in this paper, modifies the computational dependencies in order to compute the models more in parallel while ensuring that computational results are appropriately synchronized. We evaluate the method in a high performance computing grid using the Brazilian Regional Atmospheric Modelling System (BRAMS). The experimental results show a reduction of up to $71 \%$ in the time to deliver local meteorological information with this approach. The approach is sufficiently general that it can be useful with other large distributed applications.
\end{abstract}

\section{KEYWORDS}

Grid Computing, High Performance Computing, Numerical Weather Prediction Models, Workflows.

\section{INTRODUCTION}

The modern atmospheric numerical forecast (weather, climate and environment) represents a scientific breakthrough in the field of meteorology. Numerical Weather Prediction (NWP) models are complex programs that represent the movement and the physical processes of the atmosphere through mathematical equations and therewith predict their evolution [1]. It started with the development of the Atmospheric General Circulation Models (AGCM) to predict atmosphere and ocean phenomena [2]. NWP models use current weather conditions (observational data, satellite image derived data, and models generated by previous days) as input, so they can predict the weather for several days in advance with a high degree of confidence. As these models permit free choice of the particular geographical area and spatial resolution, forecasts can be run as frequently as required.

NWP generation is computationally expensive since it deals with the execution of complex calculations, which increase in number for finer spatial and temporal resolutions [3, 4]. The calculations become more complex as a result of improvements in computing chemical and physical processes in conjunction with the need to manipulate huge meteorological datasets. As a result, the main meteorological centers around the world execute global and regional area models, DOI: 10.5121/ijgca.2012.3401 
generating outputs at global or regional scales. In turn, regional meteorological centers execute local area forecast models to generate more accurate forecasts with high resolution for a specific geographical area, which are more appropriate for certain users and other applications.

The European Center for Medium-Range Weather Forecasts (ECMWF) produces timely global short-term forecasts to provide Member and Co-operating States (33 countries) with boundary conditions for their limited area models [5]. The National Meteorological Services from these countries use ECMWF's results for input to their own regional models (e.g. on a continental or national scale), to produce high-resolution forecasts for early warning of potentially damaging severe weather [6].

The National Center for Environmental Prediction (NCEP), an arm of the NOAA's National Weather Service (NWS), provides a wide variety of national and international weather guidance products to National Weather Service field offices, government agencies, emergency managers, private sector meteorologists, meteorological organizations, and societies throughout the world [7]. NCEP is a critical national resource in global/national weather prediction and the starting point for nearly all weather forecasts in the United States [8].

The Center for Weather Forecast and Climate Studies (CPTEC), from the National Institute for Space Research (INPE) in Brazil, executes various types of atmospheric numerical models daily, producing weather forecast products for Brazil and South America [9]. The INPE/CPTEC delivers meteorological information as raw model outputs or web-based products, derived from these models [10]. Regional weather centers in Brazil and South America use the raw model outputs to produce high-resolution local area weather forecasts using limited area models.

User interests in the information provided by NWP models are sensitive to the spatial resolution and geographical area. Output from these models are used for a variety of purposes: meteorologists have interest in the images or raw data from model for weather analysis, agriculturists are interested in precipitation distribution over specific areas in order to manage crops, and hydrologists use the output as input to hydrological models for water management. Transportation, energy consumption, construction, and tourism also have particular interest in weather conditions. A very important application is to provide timely warning of weather extremes, since anomalous weather events can cause impact on many lives and cause significant financial losses. The warnings that result from this additional guidance can enable great savings of both life and property [2].

In Brazil, these high resolution NWP models are dependent on the outputs of lower resolution NWP models, where "lower" resolution means coarser granularity in the computational model, e.g., a $20 \mathrm{~km} \times 20 \mathrm{~km}$ resolution is "lower" than a $5 \mathrm{~km} \times 5 \mathrm{~km}$ resolution. The higher the resolution in a computational model means that there are greater computational requirements for the same geographical region [11]. Hence, a regional model would have a lower resolution than a more localized model, but cover a larger geographical area, e.g. a continent. Regional models are computed at INPE/CPTEC, and the outputs from these models are then used as input by various local models at universities, meteorological institutions, etc. where the local models are computed. The outputs from the regional models are distributed to these institutions over nondedicated Internet links connecting institutions, which can experience delays in the transmission of meteorological data due to data traffic variation during the day.

The current workflow, therefore, entails the execution of a regional model at INPE/CPTEC and distributing those results to computational centers throughout Brazil which then run local models on local computing resources. Even without delays in the network, this takes time to generate local models. In this paper we developed an approach to reduce the overall workflow time to produce the local NWP models. The approach assumes that the underlying computational 
International Journal of Grid Computing \& Applications (IJGCA) Vol.3, No.4, December 2012

infrastructure forms a loosely coupled grid of high performance computing clusters. This is not far from the existing computational environment in Brazil for NWP models, but does require somewhat more coordination and cooperation of the individual computing resources. The approach does not involve changes to the NWP software, but does involve changing the workflow, movement of results and additional software to increase parallel computation as well as handle some synchronization between computations.

The current research on NWP models and grid environments has focus on single-user [12] or collaboration $[13,14]$ use. Since NWP models are typically tightly coupled MPI applications, the research in this area exploit the use of ensemble techniques [15, 16, 17], which involves multiple simulations of the same NWP model using different initial conditions. In this case the executions are independent and asynchronous.

The remainder of the paper is structured as follows. In Section 2 we describe the Brazilian Regional Atmospheric Modelling System (BRAMS) and its workflow. We introduce the new approach to synchronize local and regional scale simulations and the submission mechanisms in a multicluster environment in Section 3. We present the results for this methodology in Section 4, the discussion in Section 5 and conclusions in Section 6.

\section{The BraZilian Regional Atmospheric Modelling System}

The Brazilian Regional Atmospheric Modelling System (BRAMS) is a version of the Regional Atmospheric Modelling System (RAMS) [18] tailored to tropical regions for production use by the regional meteorological centers and research universities in Brazil [19]. The BRAMS (http://brams.cptec.inpe.br) is a multipurpose numerical weather model with explicit parallelization, well suitable for HPC clusters, and designed to simulate atmospheric circulations in operational forecasting and atmospheric research. It serves a broad range of applications and provides outputs of weather forecasts of granularity from meters to thousands of kilometres.

The BRAMS simulation uses analysis and boundary conditions as input data derived from a lower spatial resolution model. A RAMSIN parameter definition file contains all parameterization related to a specific simulation [20]. The BRAMS model execution generates files at certain intervals of time, representing the weather forecast for the next hours/days. These files contain arrays of data and each array is associated with a specific physical variable, atmospheric level and instant of time. Local models use this information as input, with a similar operation but with higher spatial resolution and smaller geographic area.

The BRAMS weather forecast generation in meteorological centers has a completely automatic process, which executes on a daily basis. Figure 1 shows the five stages of the BRAMS forecast workflow. The first stage includes download of input data (sea surface temperature, soil type, topography and state of the atmosphere) and conversion of the state of the atmosphere data to the RAMS standard input format for pressure coordinates and observational data (dp files; RALPH format (http://atmet.com/html/docs/data/ralph2.pdf)).

In the next three stages, the BRAMS uses a single text file in a Fortran namelist format (RAMSIN namelist). Each of these stages (see Figure 1) is responsible for different processing:

- MAKESFC: During this stage, the data in the global data files (sea surface temperature, soil type and topography) is converted to meet the resolution and area required for the regional modes as defined in the namelist \$MODEL_GRIDS (in RAMSIN).

- MAKEVFILE: This stage converts the data in the global initial and boundary conditions files to the appropriate resolution. This is performed by "cutting" the dp files in to the 
International Journal of Grid Computing \& Applications (IJGCA) Vol.3, No.4, December 2012

desired geographical areas, grids and time intervals, as defined in the namelist \$MODEL_GRIDS (in RAMSIN).

- INITIAL: This stage entails the core model execution, which can be serial or parallel (MPI-based). It uses input the data files originating from the previous two stages: topography, sea surface temperature, vegetation cover, initial and boundary condition files. The simulation generates two kinds of output data files: history and analysis. This stage provides a meteorological forecast for the desired limited area and time period. The BRAMS simulation saves history files on disk, which are required for the BRAMS restart (checkpoint files).

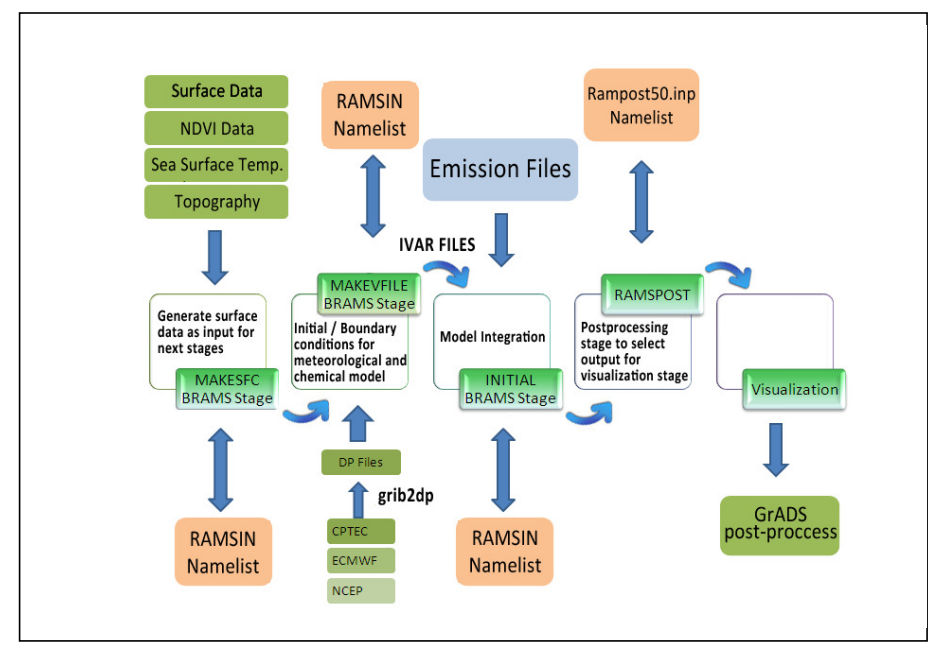

Figure 1. BRAMS workflow.

The post-processing stage includes output format conversion and a visualization process:

- Format conversion: A file format conversion program (RAMSPOST) converts the model output format (RALPH) to GrADS format (http://www.iges.org/grads/gadoc/aboutgriddeddata.html), based on rules established by RAMSPOST namelist file (Rampost50.inp). It also generates metadata information about the file contents.

- Visualization process: This stage uses GrADS [21] to generate a few diagnostic meteorological charts to allow the evaluation of the simulation results, generation of weather prognostics by meteorologists, and publication on meteorological centers websites.

The local scale simulations use the analysis and boundary conditions resulting from the BRAMS regional scale simulation. Thus, the initiation of the local scale simulation depends on the completion of the regional scale simulation and the transfer of all regional scale simulation outputs (Figure 2). 
International Journal of Grid Computing \& Applications (IJGCA) Vol.3, No.4, December 2012

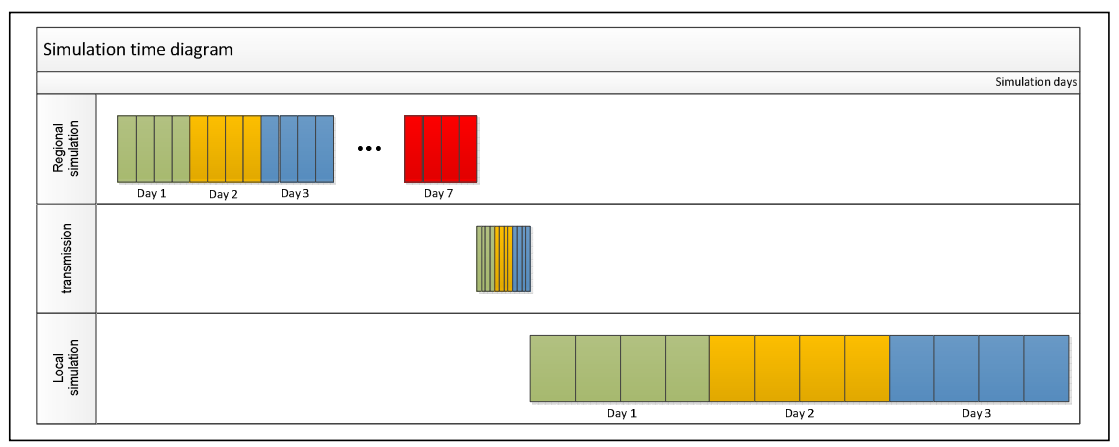

Figure 2. Time steps of BRAMS meteorological delivery process.

As presented in Figure 2, the BRAMS regional scale simulation generates weather forecast for seven days ahead, at six hour intervals (four time periods per forecast day). The regional meteorological centers download this data and execute local scale simulations to generate weather forecasts for three days ahead, with six hour intervals (four time periods per forecast day). The regional scale simulation generates twenty eight (28) files, but local scale simulation uses only the first twelve (12) of the BRAMS forecast files.

In order to address numerical stabilization, NWP models divide each six hour interval into small time steps because they can not calculate large changes in the atmospheric state for the entire six hour period. Increasing model resolution requires more and smaller time steps and, consequently, more computational power [22]. The regional and local scale models use 720 and 1440 time steps of $30 \mathrm{~s}$ and $15 \mathrm{~s}$, respectively, to generate a weather forecast output for every 6 hours. The sum of the regional scale model execution time, regional model data transmission time and local scale model execution time constitutes the total processing time for delivery of a local forecast and is given by equation (1).

Time $=\left(d_{R} \times t_{s R} \times t_{\text {Rexec }}\right)+\left(d_{R} \times t_{s R} \times t_{R \text { trans }}\right)+\left(d_{L} \times t_{s L} \times t_{\text {Lexec }}\right)$

Here $d_{R}, t_{s R}$ and $t_{\text {Rexec }}$ represent the number of days, time steps and execution time for a regional scale simulation, respectively; $t_{R t r a n s}$ is the transmission time of each regional simulation output (assuming that each is roughly the same time); and $\mathrm{d}_{\mathrm{L}}, \mathrm{t}_{\mathrm{sL}}$ and $\mathrm{t}_{\mathrm{Lexec}}$ represent the number of days, time steps and execution time for a local scale simulation, respectively.

\section{THE GRID APPROACH}

The new approach to the computation of regional and local models is to flow partial results (as manifested by the output data files) as they are produced, from the regional model to local models, and to execute the regional and local weather simulation in a parallel and loosely synchronized manner; this is illustrated in Figure 3. The objective is to reduce the time for the local models to start producing forecasts, which should be possible since the local model simulation can start execution one some of the regional files are produced, rather than waiting until the full regional model simulation ends.

The local meteorological centers only need a three day forecast from the regional scale model simulation since they normally produce local weather forecast for three days ahead (delivering output forecasts four times per day). Considering that with this approach, the local scale model execution starts sooner than if it had to wait on the completion of the regional scale model execution, the estimated total execution time is:

Time $=t_{\text {Rexec }}+t_{\text {Rtrans }}+\left(12 \times t_{\text {Lexec }}\right)$ 
International Journal of Grid Computing \& Applications (IJGCA) Vol.3, No.4, December 2012

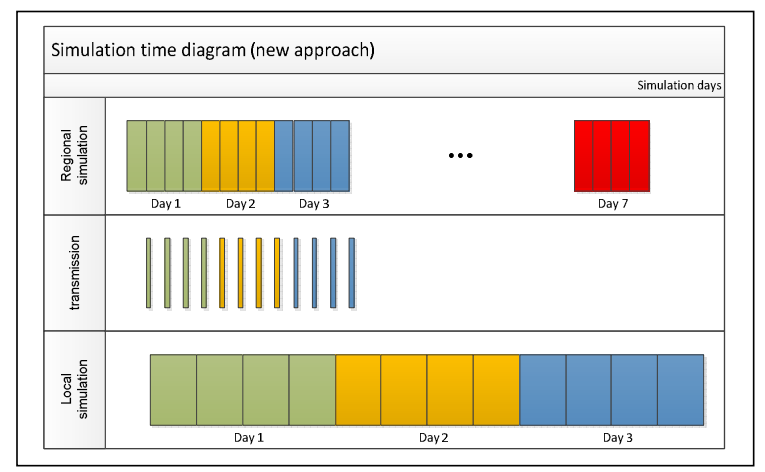

Figure 3. New regional and local scale simulation approach.

As presented before, the BRAMS simulation workflow has serial and parallel processes. For this new approach, we modify the original workflow by grouping all input serial process into a preprocessing phase, all output serial processing in a post-processing phase and execute the model execution as a parallel process. The intent is that as the regional model produces results, in this case a data file representing a meteorological forecast for a 6 hour simulated time, then that file could be moved to local processing centers and then the BRAMS simulation for the local model could, at least in principle, begin execution.

While conceptually this seems straightforward, operationally it is more complex. First, the regional and local simulations are separate computations, possibly running on different kinds of hardware. Apart from the use of the data files from the regional simulation, they are independent. Second, if we assume a grid with multiple computing facilities, they may or may not be tightly integrated. Typically, such facilities might operate relatively autonomously, each having their own resource allocation and job submission system. There could be some meta-scheduler or overarching workflow management system, though is unlikely, which means that there is no straightforward way to schedule the start of jobs on two different systems. Finally, there needs to be some assurance that the local simulation only begins once "valid" data has been acquired.

Figure 4 presents the regional scale workflow (RSWF). The pre-processing phase converts GRIB format results of the INPE/CPTEC AGCM to dp format, using GRIB2DP program, and executes the MAKESFC and MAKEVFILE stages of the BRAMS. Model execution consists of the BRAMS simulation itself (INITIAL stage). The post-processing phase occurs in parallel with the generation of regional model files. It is during this phase that the verification of the BRAMS model output is done on a regular basis by examining the six-hour simulation outputs. Once these verified outputs are available, the post-processing phase converts the BRAMS simulation outputs to GrADS format and generates regional scale meteorological field images. 
International Journal of Grid Computing \& Applications (IJGCA) Vol.3, No.4, December 2012

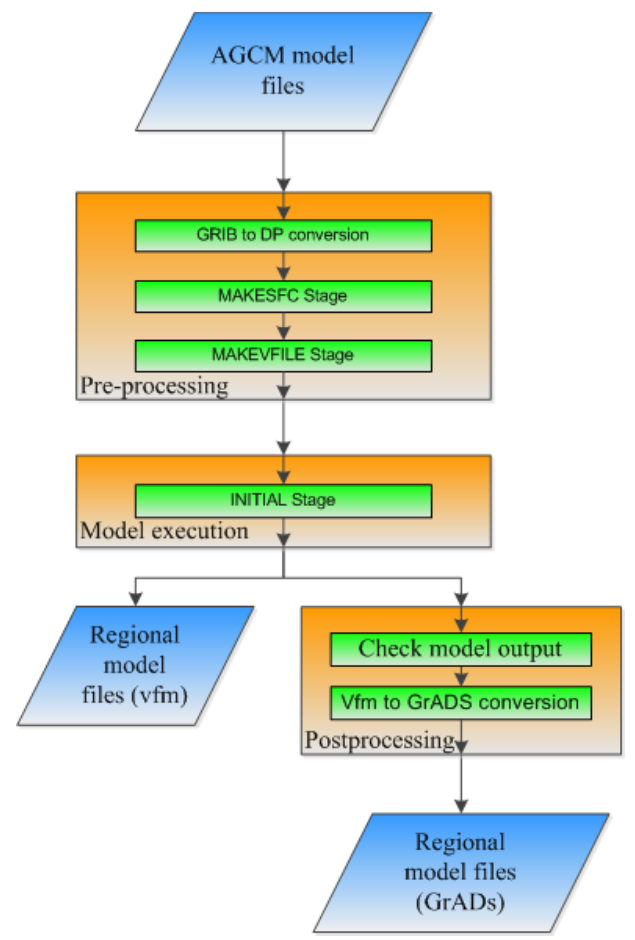

Figure 4. Regional scale workflow.

The schedule of delivering regional scale forecast data is well known by Brazilian weather centers. We developed a data transfer mechanism (Figure 5) that starts automatically based on this schedule. It downloads (when available) the regional model outputs according to the model information list (file containing the name of the files for download).

After downloading the file, this mechanism verifies that its size matches the correct size. It serves to verify if regional simulation has finished the data generation of each 6 hour forecast data, producing a valid output. The process continues up to the detection and download of the last regional scale forecast output (for three days ahead). Additionally, it monitors which files were received, looking for the presence of the analysis results and the first forecast output (the first forecast for the first 6 hours ahead). Once detected, it starts the local scale workflow independently of the data transfer mechanism. 
International Journal of Grid Computing \& Applications (IJGCA) Vol.3, No.4, December 2012

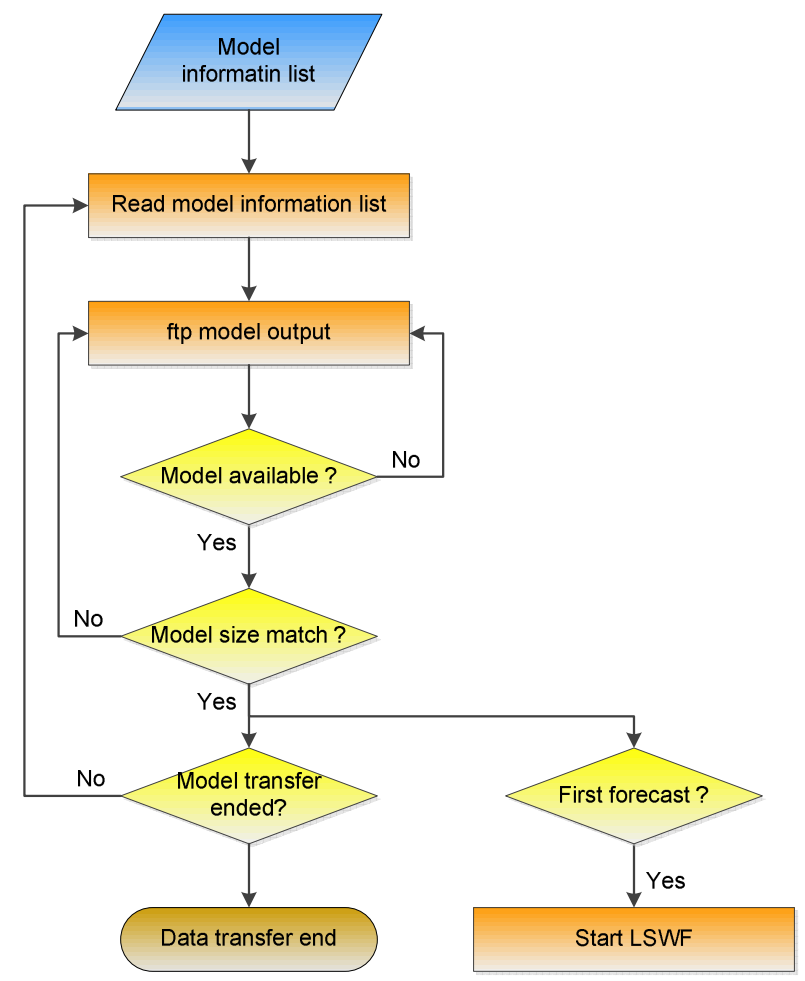

Figure 5. Data transfer mechanism.

Figure 6 shows the local scale workflow (LSWF) that uses the regional model files acquired by the data transfer mechanism and starts only when the regional analysis and the first simulation output (six-hours ahead) are available. The pre-processing phase converts regional scale model outputs from GrADS format to dp format, using GRIB2DP, and executes the MAKESFC and MAKEVFILE stages of the BRAMS. The model execution phase consists of the BRAMS simulation itself (INITIAL stage), which generates the local model files. In parallel, the local post-processing phase executes and verifies the BRAMS model output on a regular basis looking for the six-hour simulation outputs. Once it finds a such an output, it converts the BRAMS simulations outputs to the GrADS format and generates local scale meteorological field images. 


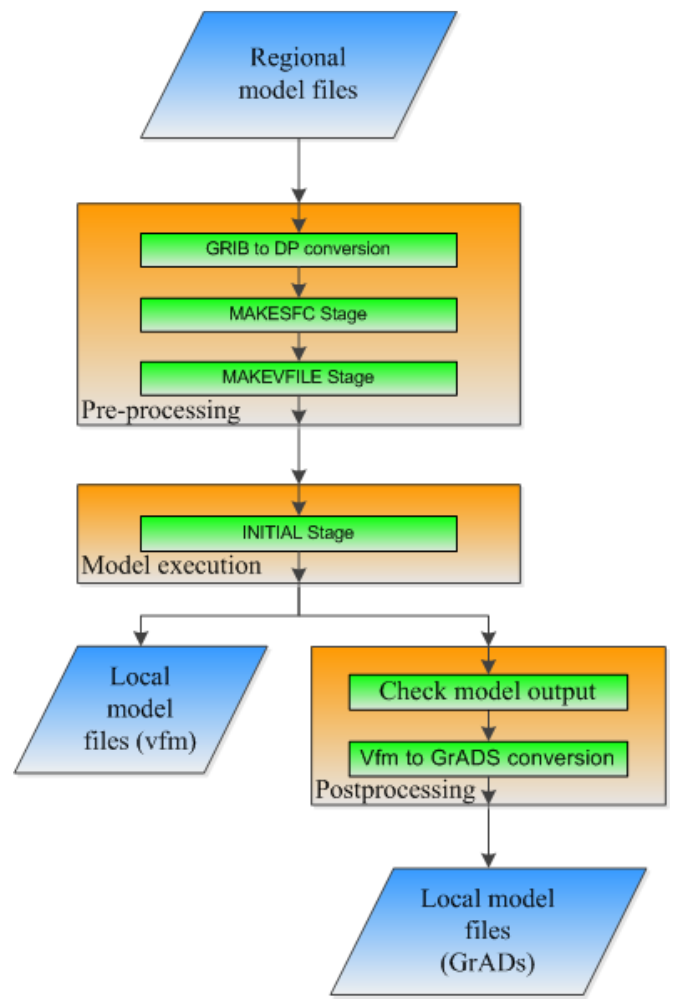

Figure 6. Local scale workflow.

The RSWF is subject to interruptions that may be caused by hardware problems, software issues or absence of input data. As a consequence, the local workflow is affected, interrupting the execution of the local simulation. As previously mentioned, the BRAMS has mechanisms to restart the simulation from the point where it was interrupted. However, this feature was not used in this work and it should be the subject of future work.

\section{EXPERIMENTAL RESULTS}

The technique of executing an atmospheric model to generate a higher resolution forecast using a lower resolution forecast as input is called "downscaling" [23]. We applied this technique in our experiment to generate regional and local scale forecasts, executing two instances of BRAMS in parallel and synchronized.

We evaluate the original and the new approach by comparing the delivery time (the BRAMS model execution at local and regional scale, and transfer time).

\subsection{Experimental Environment}

The Shared Hierarchical Academic Research Computing NETwork (SHARCNET: www. sharcnet.ca) is a high performance computing consortium of 17 academic and research organizations in Ontario. The SHARCNET grid has a number of different HPC clusters, located in different locations and interconnected using dedicated high speed networks. The HPC clusters of SHARCNET have varying interconnection networks and types of processors. Each cluster has its own resource manager and scheduler. However, the overall SHARCNET environment supports a single sign-on, which uses the Lightweight Directory Access Protocol (LDAP) to 
control account management. As a result, a researcher can access any of the systems through a single account and since a user account belongs to a global storage system, so codes compiled in a user account can be executed on any HPC cluster from SHARCNET grid. Each cluster has a job scheduler that performs job submission [24].

In the current practice in Brazil, the start of the execution of the local scale model depends on the generation of all outputs from the regional scale model. The non-dedicated RNP ("Rede Nacional de Pesquisa e Ensino") communication network provides the means to the transfer of meteorological information from INPE/CPTEC to local centers. RNP is Brazil's NREN (National Research and Education Network) and provides advanced network services to the higher education and research community [25].

For our experiments, we make use of three clusters within the SHARCNET grid:

- Whale (3072 cores): A 768 nodes HP Linux cluster with Linux CentOS and OpenMPI 1.4.2, two Opteron Dual-Core processor@2.2GHz (GigabitEthernet), and 4GB memory.

- Narwhal (1068 cores): A 267 nodes HP Linux cluster with Linux CentOS and OpenMPI 1.4.2, two Opteron Mono-Core processor@2.2GHz (Myrinet), and 8GB of memory.

- Saw (2688 cores): A 336 nodes HP Linux cluster with Linux CentOS and OpenMPI 1.4.2, two Xeon Quad-Core processor@2.83GHz (Infiniband/DDR), and 16GB of memory.

In this experiment we used the BRAMS model to simulate this weather forecast operation at the national center and to produce regional and local area weather forecast files. We used combinations of $8,16,24$ and 32 cores to execute local and regional weather simulations. We chose whale for local area weather simulations and whale, narwhal and saw for regional area weather simulations. The University of Guelph hosts narwhal and the University of Waterloo hosts whale and saw, which are $29 \mathrm{~km}$ apart.

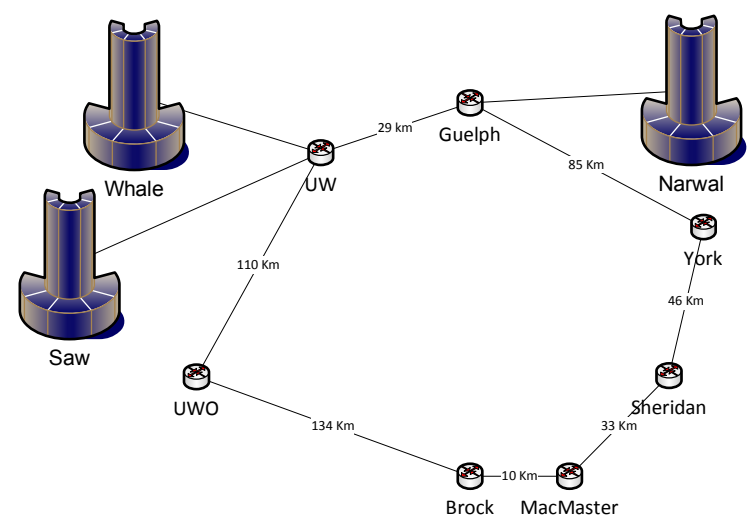

Figure 7. HPC clusters from the SHARCNET grid.

\subsection{BRAMS NWP Experiments}

We used the coarse outputs from the INPE/CPTEC AGCM model of 10/23/2010, as a starting point for the regional forecast simulation. This AGCM runs four times a day (00, 06, 12 and 18 UTC), producing analyses and numerical weather forecast data in GRIdded Binary (GRIB) and GrADS format, for fifteen (15) days ahead in T162L28 resolution. Here, T refers to spectral 
truncation type (triangular) in zonal wave 62 (resolution of $100 \times 100 \mathrm{~km}$ ) and L refers to the number of vertical levels (28 levels) [26]. Figure 8 shows one of the global scale output fields (temperature) for 24 hours ahead from 10/23/2010.

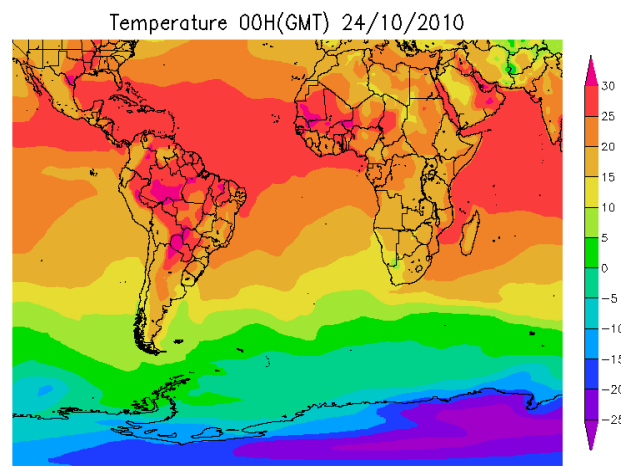

Figure 8. Global scale forecast (temperature)

Using the RSWF, we simulated a regional weather forecast over South-America for seven (7) days ahead from 10/23/2010, using the BRAMS configured for a spatial resolution of $20 \times 20 \mathrm{~km}$ and 38 atmospheric levels (a $340 \times 370 \times 38$ points matrix). The inputs, analysis and the boundary conditions for the regional scale simulation, came from initial condition and the forecast outputs of the INPE/CPTEC AGCM model execution of October $23^{\text {rd }}, 2010$.

For the regional scale simulation, the BRAMS generates analysis and forecast data for seven (7) days ahead from 10/23/2010. Figures 9 presents one of the regional scale forecast fields (temperature) produced by the RSWF, for 24 hours ahead from 10/23/2010.

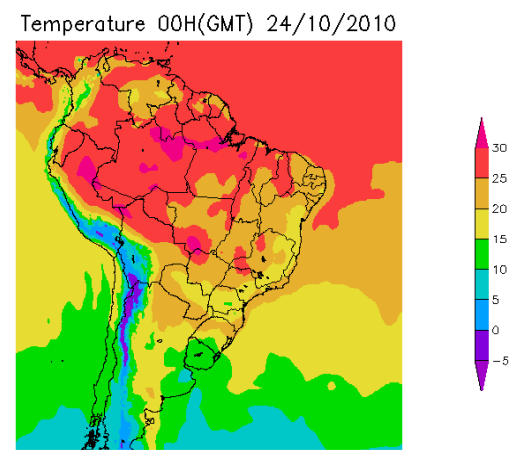

Figure 9. Regional scale forecast (temperature).

Using the local scale workflow, we simulated a local weather forecast for three (3) days ahead over the state of São Paulo, with a spatial resolution of $5 \times 5 \mathrm{~km}$ and 38 atmospheric levels (a $280 \times 250 \times 38$ points matrix). The inputs, analysis and the boundary conditions for the local scale simulation came from the initial conditions and the forecast outputs of the regional scale model execution of October $23^{\text {rd }}, 2010$.

The LSWF produces local scale analysis and three (3) days forecasts. Figure 10 presents one of the local area scale forecast fields (temperature) produced by the LSWF for 24 hours ahead from $10 / 23 / 2010$. 
International Journal of Grid Computing \& Applications (IJGCA) Vol.3, No.4, December 2012

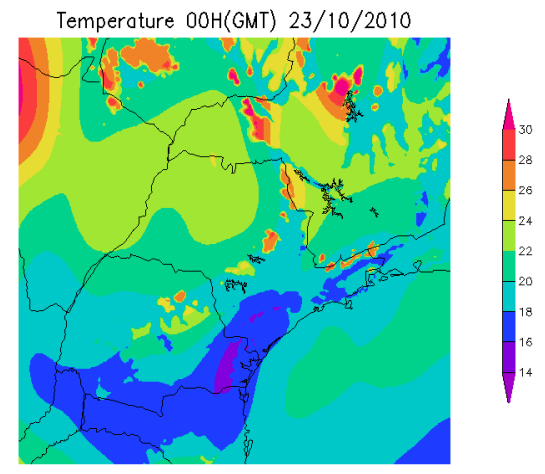

Figure 10. Local scale forecast (temperature).

Figures 11, 12 and 13 present the time for executing the regional simulation and local simulation using different number of cores, based on the original process ("Sequential" in the figures) in which the regional model is run and its outputs are then passed to local models. In the best scenario, the entire computational process, namely from the regional simulation to the production of local meteorological information, takes $24,389.3 \mathrm{~s}$ using 32 cores of saw for the regional simulation and 32 cores of whale for the local simulation. In the worst scenario, the complete production process takes $107,761.3 \mathrm{~s}$ using 8 cores of whale for the regional simulation and 8 cores of whale for the local simulation.

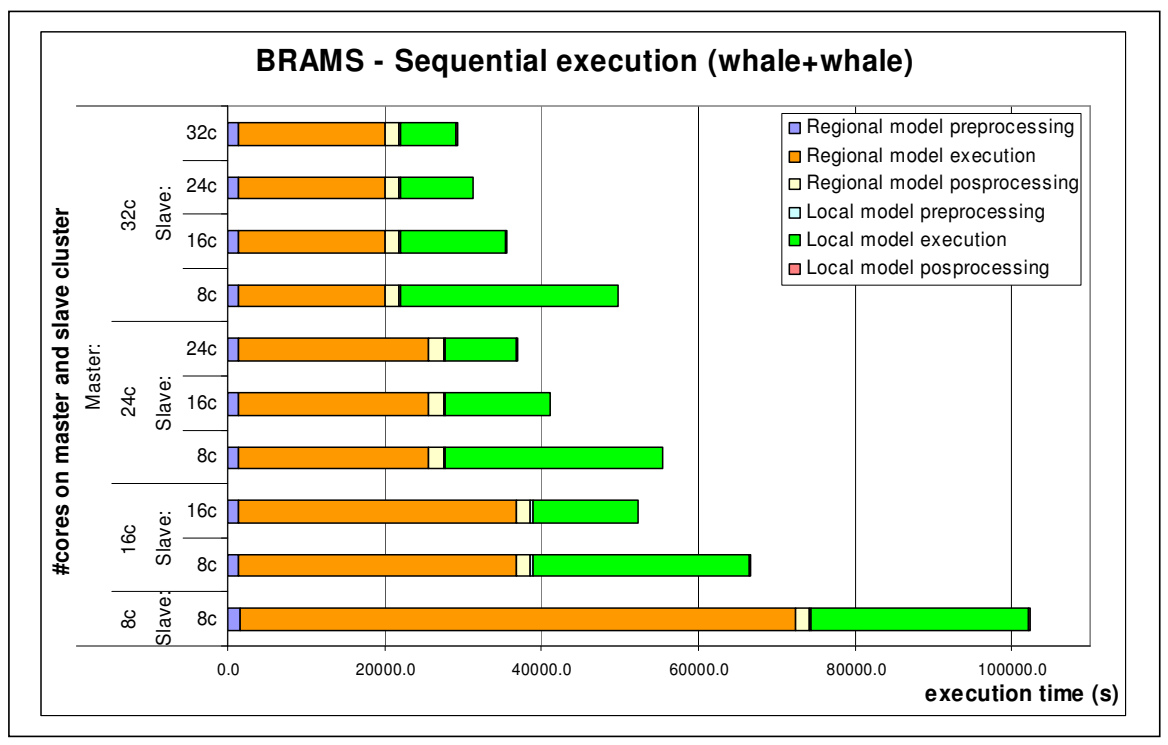

Figure 11 Sequential simulation whale/whale. 
International Journal of Grid Computing \& Applications (IJGCA) Vol.3, No.4, December 2012

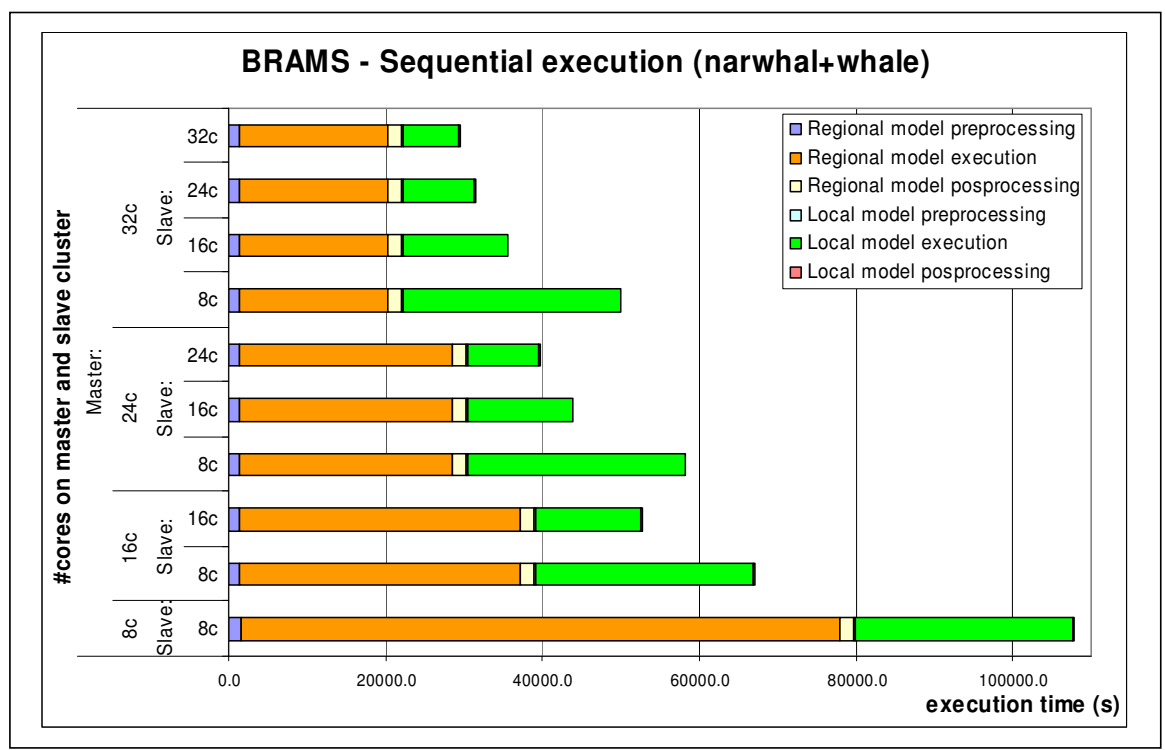

Figure 12 Sequential simulation narwhal/whale.

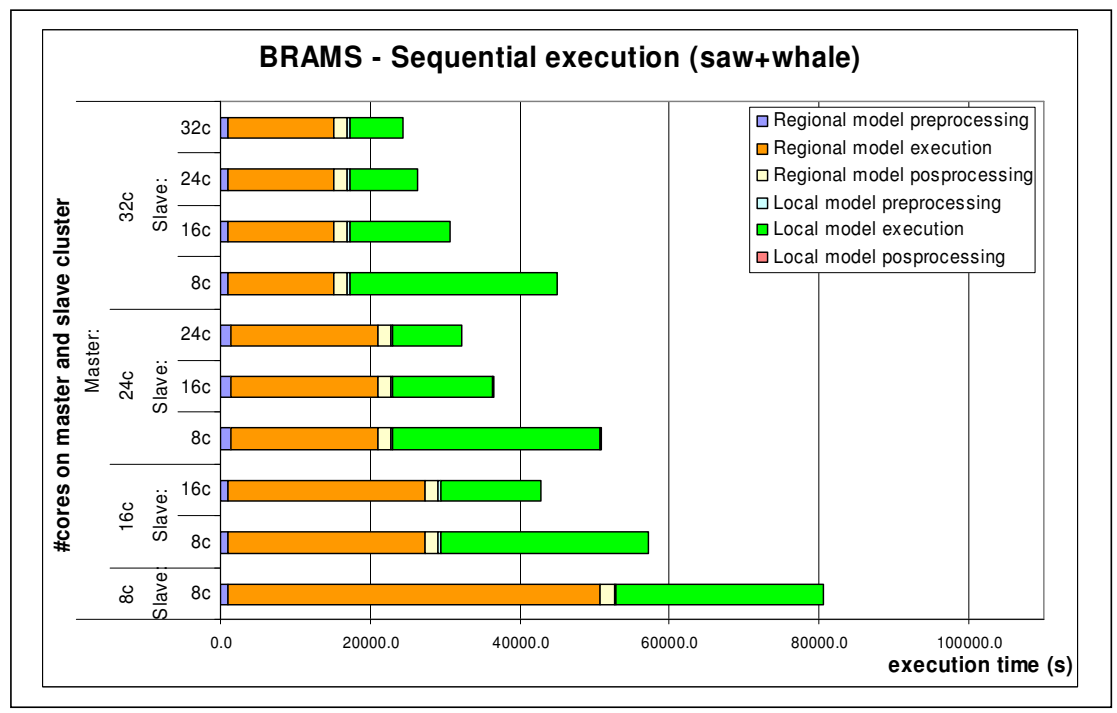

Figure 13 Sequential simulation saw/whale.

The new approach, as described, takes advantage of the fact that once the regional model has produced some data, we can begin that computation. In Figure 14, we present the total time for the complete process, that is, to production of the local meteorological information. Figure 14 also presents the percentage of reduction in time with this new approach when compared with the standard computational approach. The result is an improvement in time of between $71.0 \%$ and $34.3 \%$ when compared to the original approach. The experiment achieved the most significant reduction time when using the same number of cores for regional and local scale simulation. Increasing the number of cores for regional simulation leads to less gain in the overall production of local forecasts. 


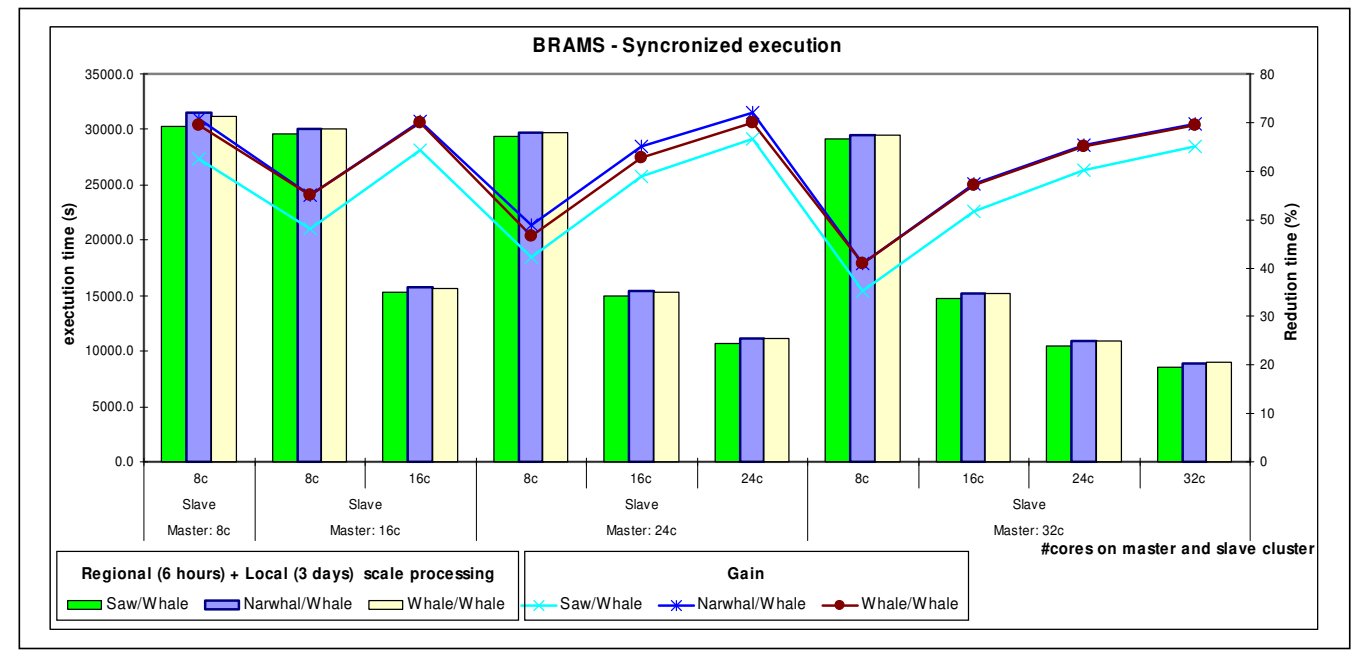

Figure 14 Results from the BRAMS synchronized execution.

The original regional model simulation generates all meteorological data outputs (535.9 MB) before any are made available for the local simulation; the new approach generates output files of 35.7 MB every six hours. The first six hours of local simulation needs the initial conditions and boundary conditions (71.5 MB).

The original approach spend 30s on average to transfer the whole regional scale output to the local scale simulation model, while the new approach uses only $2 \mathrm{~s}$ to transfer regional scale outputs for the first six hours of simulation. The data transfer time can be significant when using a busy Internet link with low bandwidth [27]. With this new approach, the reduction in the time to transfer has been reduced and so the local simulation can begin sooner resulting in an overall improvement in the time to deliver local forecasts.

\section{DISCUSSION}

The experiment, though using a dedicated network, showed significantly reduced transfer time and so would be expected to have a significant impact when using a busy shared Internet connection with low bandwidth. In the near future, the new RNP (Brazilian National Research and Education Network - NREN) network infrastructure will provide Dynamic Circuit Provisioning for Brazilian institutions, with the main purpose of delivering bandwidth on demand for applications with high demand for quality of service (QoS) [25]. In this context, this work is particularly important, since RNP connects the meteorological centers in Brazil and this new approach will permit the creation of a computational grid on demand to support weather forecast production by guaranteeing bandwidth for meteorological information data transfers. The SHARCNET grid has provided all resources to evaluate in advance this approach in a similar environment.

We also observed that during the running of the experiments that there was the occasional interruption of the local scale simulation due to demands for computational resources and the unavailability of the communication network. In meteorological centers, the meteorological operational process has priority over non-priority processes during regular operation. These processes execute in a special queue, allowing their execution to begin while placing other processes in suspension (sleep) mode. However, hardware and software failures may occur [28], preventing simulations from starting execution or new simulations may require additional computational resources. In Brazil, the National System of High Performance Computing 
International Journal of Grid Computing \& Applications (IJGCA) Vol.3, No.4, December 2012

(SINAPAD) coordinates the network of geographically distributed High Performance Computing Centers (CENAPAD). The proposed submission mechanism could be used by the meteorological centers on one of the distributed HPC clusters from SINAPAD, since they also rely on queues, to provide an additional computational power. The SINAPAD HPC systems could also be used as a contingency system. Fault-tolerance and workflow management will be addressed on our future work.

Other complex applications exist which also use distributed workflows, and require a large number of computing nodes, which are typically unavailable within one organization. The Disaster Simulation system [29] assesses the evolution and impact of various physical phenomena, in domains such nuclear and environmental science. Aircraft industries use large scale multi-physics applications (aircraft flight dynamics simulation) to reduce the time of development [30]. The Ensemble Kalman Filter (EnKF) method is an important application in the petroleum industry for reservoir and groundwater modelling, and is used for operational monitoring and prediction [31]. The NWP model WRF ("Weather Research and Forecasting") is used for hurricane forecasting in high spatial resolution and ensemble mode [27]. Many of these applications could also make use of this approach.

\section{CONCLUSIONS}

In this paper, we proposed a new strategy to reduce the delivery time of meteorological information at local scale. The original approach had a sequential workflow, which first executes the NWP model at a regional scale at a national facility and then transmits the regional scale information to other computation centers, which finally execute the NWP at local scale. By changing the way outputs were produced and interleaving their transmission to other computation centers, we are able to start the local scale weather simulation sooner. The new approach monitors the regional scale output to detect the occurrence of the first regional forecast output. Then the local scale weather simulation can be started.

We evaluated the new approach by executing local and regional scale simulations on three different configurations HPC clusters of the SHARCNET grid: on the same cluster (whale), on two sets of different clusters (narwhal and whale, and saw and whale). This strategy showed a significant reduction (up to $71 \%$ ) in time to deliver local meteorological information by synchronizing local scale weather simulation with regional scale weather simulation.

In the near future, RNP will provide Dynamic Circuit Provisioning for Brazilian institutions on its new backbone network. By guaranteeing QoS and working similarly to SHARCNET links in a predetermined window time, the new RNP infrastructure will allow the efficient use of the methodology presented in this paper. The NWP application may fail, due to the lack of input data or the unavailability of computational infrastructure. In the future, we will investigate fault tolerant mechanisms to address this problem.

\section{ACKNOWLEDGEMENTS}

This work was made possible by FAPESP (process: 2010/05823-7) financial support and the facilities of the Shared Hierarchical Academic Research Computing Network (SHARCNET: www.sharcnet.ca) and Compute/Calcul Canada. We would like to thank Baolai Ge, from SHARCNET, and Luiz Flavio Rodrigues, from INPE for their support 
International Journal of Grid Computing \& Applications (IJGCA) Vol.3, No.4, December 2012

\section{REFERENCES}

[1] Pielke, Roger A. (2002). Mesoscale Meteorological Modeling. Academic Press. p. 65. ISBN 0-12554766-8

[2] P. Lynch, "The origins of computer weather prediction and climate modelling," in: Journal of Computational Physics, Volume 227, Issue 7, Predicting weather, climate and extreme events, pp. 3431-3444, 2008.

[3] Ollinaho, P., Laine, M., Solonen, A., Haario, H., \& Järvinen, H. (2012). NWP model forecast skill optimization via closure parameter variations. Quarterly Journal of the Royal Meteorological Society.

[4] Jourdain, N. C., Marchesiello, P., Menkes, C. E., Lefèvre, J., Vincent, E. M., Lengaigne, M., \& Chauvin, F. (2011). Mesoscale simulation of tropical cyclones in the South Pacific: climatology and interannual variability. Journal of Climate, 24(1), 3-25.

[5] Bengtsson, L. (1981). Work of the European center for medium range weather forecasts. Ocean Management, 7(1), 99-105.

[6] A. Pearson, "User guide to ECMWF forecast products", In: Meteorological Bulletin M3.2, ECMWF, Reading, United Kingdom, 115 pp, 2001.

[7] NCEP (2009) National Centers for Environmental Prediction Strategic Plan 2009-2013.

[8] NCEP "National Centers for Environmental Prediction (NCEP)". [Online]. Available: http://www.ncep.noaa.gov/about/ [Accessed: 24 Nob. 2012].

[9] Prado, A. F. B. D. A. (2011). A short history of the academic activities at the Brazilian National Institute for Space Research. Journal of Aerospace Technology and Management, 3(1), p-5.

[10] CPTEC, "Centro de Previsão de Tempo e Estudos Climáticos (CPTEC)". [Online]. Available: http://www.cptec.inpe.br/ [Accessed: 18 Nov. 2012].

[11] Zhou, L. J., Liu, Y. M., \& Bao, Q. (2012). Computational performance of the high-resolution atmospheric model FAMIL. Atmos. Oceanic Sci. Lett, 5, 355-359.

[12] Souto, R. P., Avila, R. B., Navaux, P. O. A., Py, M. X., Maillard, N., Diverio, T. A., ... \& Almeida, E. S. (2007, May). Processing mesoscale climatology in a grid environment. In Cluster Computing and the Grid, 2007. CCGRID 2007. Seventh IEEE International Symposium on (pp. 363-370). IEEE.

[13] Ma, T., Ge, J., Cao, H., \& Wang, Y. (2010, November). Design and Implementation of Virtual Resources Management in Meteorology Grid. In Grid and Cooperative Computing (GCC), 2010 9th International Conference on (pp. 58-63). IEEE.

[14] Plale, B., Droegemeier, K. K., \& Mattocks, C. (2010, January). The LEAD Gateway II: A Hardened, Persistent Community Resource for Meteorological Research and Education. In 26th Conference on Interactive Information and Processing Systems.

[15] Strazdins PE, Kahn M, Henrichs J, Pugh T, Rezny M (2011) Profiling Methodology and Performance Tuning of the Met Office Unified Model for Weather and Climate Simulations, in 25th IEEE International Parallel and Distributed Processing Symposium (PDESC-11 Workshop). Anchorage: IEEE..

[16] Kotroni, V., Floros, E., Lagouvardos, K., Pejanovic, G., Ilic, L., Zivkovic, M.: Multi-model multianalysis ensemble weather forecasting on the Grid for the South Eastern Mediterranean Region. Earth Sci. Inform. 3, 209-218 (2010)

[17] Fernández-Quiruelas, V., Fernández, J., Cofiño, A. S., Fita, L., \& Gutiérrez, J. M. (2011). Benefits and requirements of grid computing for climate applications. An example with the community atmospheric model. Environmental Modelling \& Software, 26(9), 1057-1069.

[18] C.J. Tremback, and R.L. Walko, "The regional atmospheric modeling system (RAMS): Development for parallel processing computer architectures”, in: 3rd RAMS Users' Workshop, 1997, Echuca, Victoria, Australia, 1997.

[19] S.R.M. Barros, "Towards the RAMS-FINEP parallel model: load balancing aspect", in: Proceedings of the 8th ECMWF - Workshop on the Use of Parallel Processors in Meteorology, Reading, UK, 1998.

[20] A.L. Fazenda, D.S. Moreira, E.H. Enari, J. Panetta and L.F. Rodrigues, "First time user's guide (BRAMS v. 3.2)", Cachoeira Paulista, 24p., 2006.

[21] IGES, GrADS: grid analysis and display system. [Online]. Available: http://www.iges.org/grads/ [Accessed: 30 Nov. 2012].

[22] S. Al-Yahyai, Y. Charabi, and A. Gastli, "A Review of the use of Numerical Weather Prediction (NWP) Models for wind energy assessment”, in: Renewable \& Sustainable Energy Reviews (RSER), Vol. 14, No. 9, pp. 3192-3198, 2010. 
International Journal of Grid Computing \& Applications (IJGCA) Vol.3, No.4, December 2012

[23] C.L. Castro, R.A. Pielke and G. Leoncini, "Dynamical downscaling: Assessment of value retained and added suing the regional atmospheric modeling system (RAMS)", J. Geophys. Res., 110 , D05108, 2005.

[24] M.A. Bauer, "High performance computing: the software challenges", in: PASCO '07: Proceedings of the 2007 international workshop on Parallel symbolic computation. New York, NY, USA: ACM, pp. $11-12,2007$.

[25] M.A. Stanton, "RNP experiences and expectations in future Internet research and development", in: Tronco, T.R. (ed.), New Network Architectures: The Path to the Future Internet, Springer, 2010.

[26] J.P. Bonatti, "Modelo de circulação geral atmosférico do CPTEC”, in: Climanálise Especial (10 anos Edição Especial), 5 pp., 1996, Centro de Previsão de Tempo e Estudos Climáticos (CPTEC), Cachoeira Paulista, Brazil. [Online] Available: www6.cptec.inpe.br/products/climanalise/cliesp10a/bonatti.html [Accessed: 30 Nov. 2012].

[27] S.M. Sadjadi, L. Fong, R.M. Badia, J. Figueroa, J. Delgado, X.J. Collazo-Mojica, K. Saleem, R. Rangaswami, S. Shimizu, H.A.D. Limon, P. Welsh, S. Pattnaik, A. Praino, D. Villegas, S. Kalayci, G. Dasgupta, O. Ezenwoye, J.C. Martinez, I. Rodero, S. Chen, J. Muñoz, D. Lopez, J. Corbalan, H. Willoughby, M. McFail, C. Lisetti, and M. Adjouadi, "Transparent grid enablement of weather research and forecasting”, in: Proc. 15th ACM Mardi Gras conference, Baton Rouge (EUA), 2008.

[28] Das, A. and De Sarkar, A. On Fault Tolerance of Resources. in Computational Grids. International Journal, 3.

[29] T.B. Ionescu, A. Piater, W. Scheuermann, E. Laurien, and A. Iosup, “An Aspect-Oriented Approach for Disaster Prevention Simulation Workflows on Supercomputers, Clusters, and Grids", in: Proc. DS-RT, pp. 21-30, 2009.

[30] T. Nguyên, L. Trifan, and J.-A. Désidéri, "Resilient Workflows for Cooperative Design", in: Proc. 15th Intl. Conf. on Computer Supported Collaborative Work in Design (CSCWD2011), 2011.

[31] X. Li, Z. Lei, C.D. White, and G. Allen, "Ensemble Subsurface Modeling Using Grid Computing Technology", in: Proc. International Multi-Symposiums on Computer and Computational Sciences (IMSCCS), pp. 235-244, 2007.

\section{Authors}

Eugenio S. Almeida is a researcher at National Institute for Space Research (INPE) and was São Paulo Research Foundation (FAPESP) post-doctoral fellow at the Computer Science Department (The University of Western Ontario) in 2010-2011. He obtained a Dr. Degree (2007) in Applied Computing and a M.Sc. degree (1997) in Remote Sensing at the INPE. His research interests are grid and HPC computing, and computer networks.

Michael A. Bauer is a Professor of Computer Science at The University of Western Ontario (UWO). He was Chair of the Department (1991-1996, 20022007, 1996-2001) and Associate Vice-President Information Technology of the UWO. He was the founding Principal Investigator for SHARCNET (www.sharcnet.ca) and is currently its Associate Director. He has Ph.D. in Computer Science from the University of Toronto. His research interests include distributed computing, autonomic systems and applications, network management, and HPC systems and networks. He has published over 250

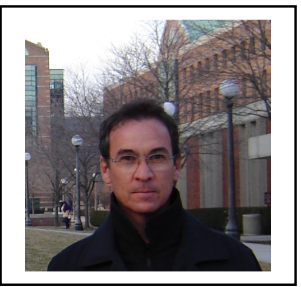
refereed articles.

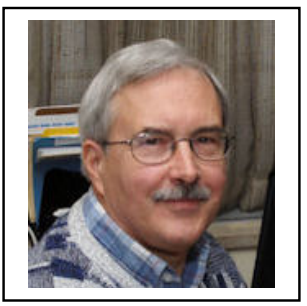

